\title{
Primary adrenal leiomyosarcoma: A case report
}

\author{
MARIA SAKELLARIOU $^{1}$, DIONYSIOS DELLAPORTAS ${ }^{1}$, EIRINI GRAPSA $^{2}$, \\ MENELAOS TZIKANOULAS ${ }^{3}$, ATHANASIOS DELLIS ${ }^{1}$, \\ THEODOSIOS THEODOSOPOULOS ${ }^{1}$ and CONSTANTINOS NASTOS ${ }^{1}$
}

\author{
${ }^{1}$ Second Department of Surgery; Departments of ${ }^{2}$ Nephrology and ${ }^{3}$ Biopathology, School of Medicine, \\ National and Kapodistrian University of Athens, Aretaieion University Hospital, 11528 Athens, Greece
}

Received May 27, 2019; Accepted December 30, 2019

DOI: $10.3892 / \mathrm{mco} .2020 .1987$

\begin{abstract}
Adrenal leiomyosarcomas are rare mesenchymal tumors of the suprarenal region that are usually diagnosed after they have reached a large size. We report the case of a 62-year-old male with an incidentally found left adrenal mass. Magnetic resonance imaging of the retroperitoneal space showed a heterogeneously enhanced mass, measuring $10 \times 8.2 \mathrm{~cm}$, with characteristics suspicious of malignancy. The patient underwent left radical adrenalectomy after the hormonal evaluation of the tumor due to the high probability of adrenocortical carcinoma. However, microscopic examination of the tumor showed a spindle cell sarcoma. Immunohistochemically the neoplastic cells were found positive for desmin and smooth muscle actin and the diagnosis of a well differentiated adrenal leiomyosarcoma was established. During follow-up the patient presented an aggressive course as he developed bone, liver and pulmonary metastases early postoperatively, which were treated with radiation therapy and chemotherapy. The patient has progressive metastatic disease while on chemotherapy 31 months after surgery.
\end{abstract}

Correspondence to: Dr Maria Sakellariou, Second Department of Surgery, School of Medicine, National and Kapodistrian University of Athens, Aretaieion University Hospital, 76 Vassilisis Sofias Ave, 11528 Athens, Greece

E-mail: marisakellariou@gmail.com

Abbreviations: LMS, leiomyosarcomas; CT, computed tomography; IV, Intravenous; MRI, magnetic resonance imaging; T1W, T1-weighted; T2W, T2-weighted; GRE, gradient recalled echo; r.r., reference range; S-DHEA, dehydroepiandrosterone sulphate; PRA, plasma renin activity; ACTH, adrenocorticotropic hormone; HPF, high power fields; SMA, smooth muscle actin; PAN CK, pan-cytokeratin; AIM, doxorubicin, ifosfamide, mesna; ${ }^{99 \mathrm{~m} T c-H D P}$, technetium Tc $99 \mathrm{~m}$ hydroxydiphosphonate; AIDS, acquired immunodeficiency syndrome; IVC, inferior vena cava

Key words: adrenal gland, neoplasia, malignant, leiomyosarcoma, adrenalectomy

\section{Introduction}

Leiomyosarcomas (LMS) constitute rare mesenchymal tumors of smooth muscle origin. LMS of the adrenal gland are retroperitoneal tumors in the suprarenal region with less than 50 cases described in the English literature. They are thought to derive from the adrenal vein and/or its branches (1). Adrenal LMS are diagnosed postoperatively after pathologic examination of the surgical specimen; they have no specific clinical or imaging characteristics nor biomarkers and are usually discovered after they have reached a large size due to compressive phenomena (2). The aim of this report is to describe the case of an adrenal LMS in a 62-year-old male patient discovered incidentally.

\section{Case report}

An asymptomatic 62-year-old Caucasian male presented to our unit for the investigation of an incidentally found left suprarenal mass, discovered during his annual sonographic follow-up for nephrolithiasis. From his medical history, except for the nephrolithiasis, the patient had diabetes mellitus type II, dyslipidaemia and an appendicectomy performed 30 years ago. Physical examination and laboratory testing revealed no abnormal findings and we proceeded with a computed tomography (CT) of the abdomen with intravenous (IV) and oral contrast agent. CT revealed a low-density mass measuring $10.3 \times 8.5 \times 8.4 \mathrm{~cm}$ originating from the left adrenal gland with minor contrast uptake, suspicious of malignancy. The subsequent adrenal protocol magnetic resonance imaging (MRI) revealed a $10 \times 8.2 \mathrm{~cm}$ mass with low-signal-intensity on T1-weighted (T1W) images and intermediately high-signal-intensity on T2-weighted images (T2W), with heterogeneous contrast uptake, without loss of signal-intensity in out-of-phase gradient recalled echo (GRE) T1W sequence (Fig. 1). The medial border of the tumor appeared to have the morphology of confluent nodules that were possibly representing infiltrated lymph nodes. The mass was rendered as highly suspicious of adrenal malignancy, most probably of adrenocortical carcinoma. We thereafter performed a chest CT scan to exclude the presence of pulmonary metastases, as well as a biochemical evaluation of the patient for hormonal hypersecretion to exclude the possibility of a hormonally active adrenal tumor. Laboratory results were as follows: $24 \mathrm{~h}$ urine 
collection for metanephrine: $62 \mathrm{mcg} / 24 \mathrm{~h}$ [reference range (r.r.) $52-341 \mathrm{mcg} / 24 \mathrm{~h}$ ], $24 \mathrm{~h}$ urine collection for normetanephrine: $367 \mathrm{mcg} / 24 \mathrm{~h}$ (r.r. 88-444 mcg/24 h), dehydroepiandrosterone sulphate (S-DHEA): $186 \mathrm{mcg} / \mathrm{dl}$ (r.r. 51.7-295 mcg/dl), plasma renin activity (PRA): $0.21 \mathrm{ng} / \mathrm{ml} / \mathrm{h}$ (r.r. $0.2-1.4 \mathrm{ng} / \mathrm{ml} / \mathrm{h}$ ), cortisol: $11.9 \mathrm{mcg} / \mathrm{dl}$ (r.r. $6.2-19.4 \mathrm{mcg} / \mathrm{dl}$ ), adrenocorticotropic hormone (ACTH): $39.0 \mathrm{pg} / \mathrm{ml}$ (r.r. 7.0-64 pg/ml), aldosterone: $5.11 \mathrm{ng} / \mathrm{dl}$ (r.r. $1.0-16 \mathrm{ng} / \mathrm{dl})$, dexamethasone suppression test: cortisol: $0.4 \mathrm{mcg} / \mathrm{dl}$. Blood sample collection was performed on September 27th, 2016.

The patient underwent left open radical adrenalectomy due to the high possibility of the tumor being a non-secretory adrenocortical carcinoma on account of imaging characteristics and its size. The extent of the resection included the tumor and all peri tumoral fat and para-aortic lymphatic tissue from the diaphragm to the level of the left renal vein. The postoperative course was uneventful and the patient was discharged on day 6 after surgery.

Histopathology. The surgical specimen was collected on October 7th, 2016. On gross pathological examination the

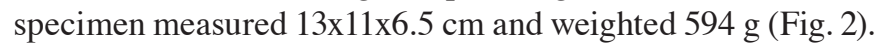
The mass had lobulated appearance, showed areas of cystic degeneration and necrosis in cross section and residual adrenal tissue was identifiable on its outer surface. The tumor was surrounded by fibroadipose tissue measuring $11 \times 8 \times 2 \mathrm{~cm}$.

Microscopic examination revealed a spindle cell tumor with intermediate nuclear atypia, significant mitotic activity [7 mitoses/10 high power fields (HPF)] and small areas of ischaemic necrosis. In certain sections the tumor was observed to have grown in the wall of a large vein, most probably the central adrenal vein and in the circumference of the mass adrenal tissue residue was identifiable. Neoplastic cells were also present in the periadrenal fibroadipose tissue with the form of discrete circumscribed nodules.

Immunohistochemistry. Tumor cells were found to be positive for desmin, smooth muscle actin (SMA) and negative for S-100 protein, synaptophysin, chromogranin, pan-cytokeratin (PAN CK), calretinin. The proliferating index Ki-67 was found to be approximately $75 \%$ (Fig. 3).

The final diagnosis of the pathological analysis was well-differentiated leiomyosarcoma of the left adrenal gland with maximum diameter being $13 \mathrm{~cm}$. There were no infiltrated lymph nodes found. What were thought to be infiltrated lymph nodes in the preoperative MRI was neoplastic infiltration of the periadrenal fibroadipose tissue in the form of satellite nodules.

Following the pathological examination report, the Multidisciplinary Oncology Council of our hospital decided the regular follow-up of the patient with image studies, which in 3 months postoperatively showed a metastatic lesion in the 10th thoracic vertebra (retroperitoneal MRI, thoracic spine MRI). The patient was then treated with radiation therapy in the region of the 9th-12th thoracic vertebrae; he received a total dose of $20 \mathrm{~Gy}$, with the daily dose being $4 \mathrm{~Gy}$. The following chest and abdomen CT performed at 7 months postoperatively were negative for pulmonary metastases, but showed metastases in the hepatic parenchyma. The patient was subsequently integrated in the Oncology Department of our Hospital for

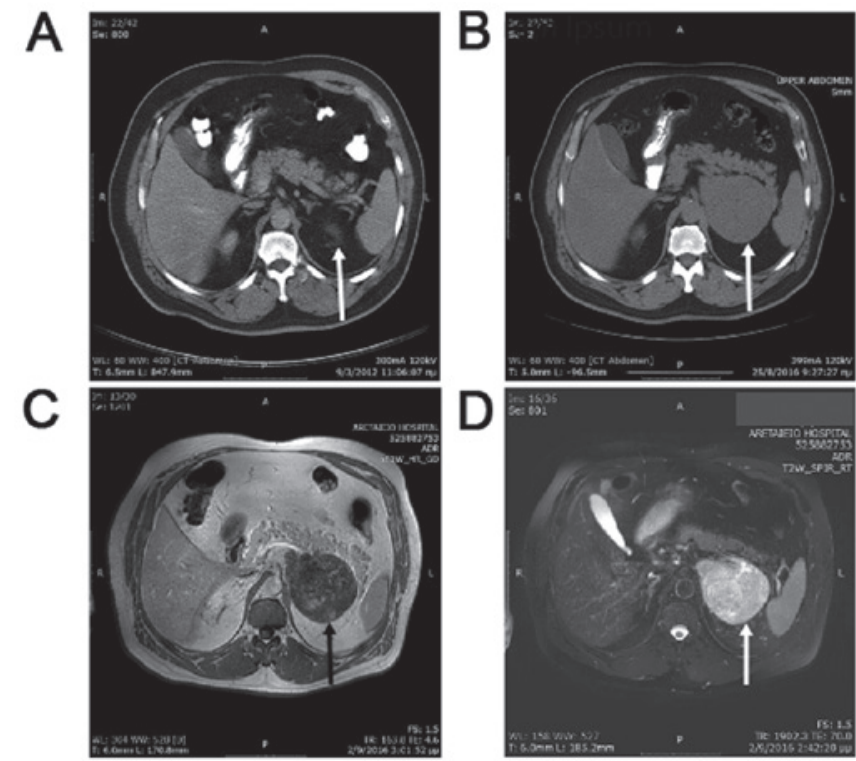

Figure 1. (A) CT scan of the patient 4 years before diagnosis showing no pathology in the area of the left adrenal (arrow). (B) CT with intravenous contrast showing a $10.3 \times 8.5 \times 8.4 \mathrm{~cm}$ mass originating from the left adrenal gland (arrow), with minor contrast uptake. (C) MRI with low signal intensity on T1-weighted images (arrow). (D) MRI with high signal intensity on T2-weighted images (arrow). CT, computed tomography; MRI, magnetic resonance imaging.

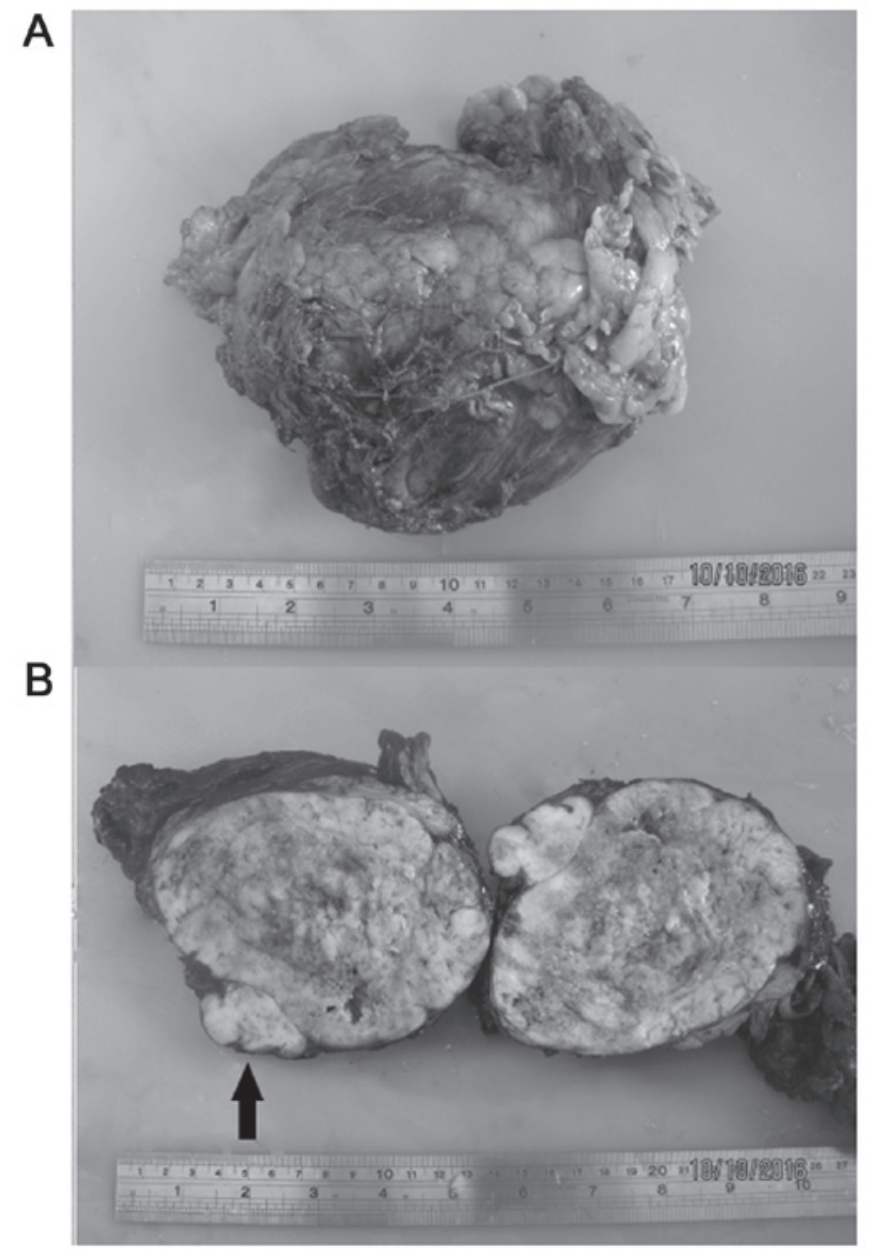

Figure 2. (A) Surgical specimen after fixation in $4 \%$ formaldehyde solution. (B) Surgical specimen sectioned and showing dense tissue with peritumoralsatellite nodules (arrow). 

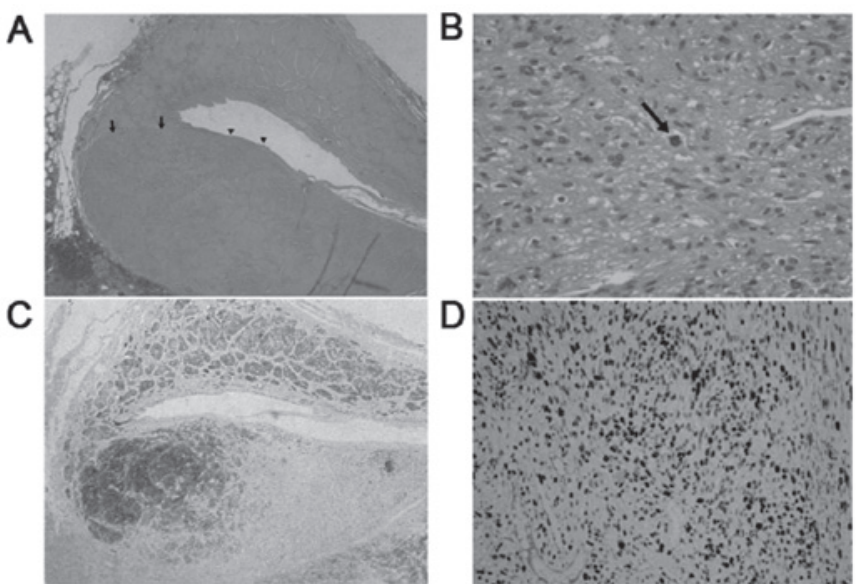

Figure 3. (A) Hematoxylin and eosin staining of the tumor revealing leiomyosarcoma tumor cells originating from the adrenal vein (arrow) and developing in its lumen (arrow heads; x20). (B) High-power-view of tumor cells on hematoxylin and eosin stain revealing abnormal mitotic figure (arrow; x200). (C) Positive Desmin staining (Di-Amino Benzidine, x20) (D) Immunohistochemical staining of cells with Ki-67 marker showing expression in over $75 \%$ of the cells (Di-Amino Benzidine, $\mathrm{x} 100$ ).

the initiation of IV chemotherapy treatment with gemcitabine and docetaxel. During this treatment the patient exhibited progression of the disease, developing pulmonary metastases 11 months postoperatively and his chemotherapy regimen was changed to AIM (doxorubicin, ifosfamide, mesna) at 16 months postoperatively, due to radiological evidence of bone, liver and pulmonary metastases growing in number and in size (chest and abdomen CT, skeletal scintigraphy with ${ }^{99 \mathrm{~m}} \mathrm{Tc}-\mathrm{HDP}$ ). The patient continues to receive IV chemotherapy treatment and is alive with metastatic disease 31 months after surgery.

\section{Discussion}

Adrenal LMS are non-functional mesenchymal tumors of the adrenal gland, with smooth muscle differentiation, which are thought to derive from the adrenal vein or its branches. They are usually found in image studies as large heterogeneous masses with no specific characteristics, which makes them undistinguishable from other adrenal tumors (3). The pathogenesis of adrenal leiomyosarcoma remains unknown, but there might be a correlation with Epstein-Barr Virus infection in patients with acquired immunodeficiency syndrome (AIDS) (4). The treatment of choice is surgical resection with negative margins, after the evaluation of the tumor secretion to exclude the diagnosis of a functional tumor. Their diagnosis is rendered after pathological examination of the specimen, which is observed microscopically as a spindle cell neoplasia with nuclear atypia or pleomorphism, high mitotic activity and necrotic foci and immunohistochemically is found positive for smooth muscle stains, although there have been described cases with pleomorphic presentation of the tumor (5).

Chemotherapy and radiation therapy have been proposed for locally advanced or metastatic disease but these treatments are yet to be proved beneficial (6). Biopsy of the tumor can provide the necessary histological diagnosis before chemo/radiation therapy if the patient is deemed inoperable (7). Adrenal LMS are usually diagnosed after they have reached a large size due to their lack of specific signs and symptoms, and in many cases the tumor is found to have extended to the inferior vena cava (IVC) upon diagnosis, or to have spread to distant organs (8). In addition, patterns of recurrent disease and metastatic spread render the behavior of this tumor rather aggressive, with longest patient survival being reported as three years after surgery (9).

In conclusion, adrenal LMS are rare tumors found in the suprarenal region, usually diagnosed having reached a large size or after having provoked symptoms due to applying pressure to adjacent structures, or by extending directly to the IVC. Their usual diagnosis in an advanced stage contributes to the poor prognosis of these patients. Their radiological characteristics are similar to those of other adrenal tumors and therefor they should be considered in the differential diagnosis of a suprarenal non-secreting mass with malignant characteristics. The gold standard of treatment is surgical excision, with chemotherapy and radiation therapy being reserved for advanced disease.

\section{Acknowledgements}

Not applicable.

\section{Funding}

No funding was received.

\section{Availability of data and materials}

The datasets used during the present study are available from the corresponding author on reasonable request.

\section{Authors' contributions}

$\mathrm{CN}, \mathrm{AD}$ and EG contributed to the conception and design of the study. MS, DD and MT acquired, analyzed and interpreted the data. MS, TT and EG drafted the manuscript. CN and TT revised the manuscript. All authors read and approved the final version of this manuscript.

\section{Ethics approval and consent to participate}

Oral patient consent was obtained for participation in this study.

\section{Patient consent for publication}

Informed consent was obtained for the publication of patient data.

\section{Competing interests}

The authors declare that they have no competing interests.

\section{References}

1. Zetler PJ, Filipenko JD, Bilbey JH and Schmidt N: Primary adrenal leiomyosarcoma in a man with acquired immunodeficiency syndrome (AIDS). Further evidence for an increase in smooth muscle tumors related to Epstein-Barr infection in AIDS Arch Pathol Lab Med 119: 1164-1167, 1995. 
2. Onishi T, Yanagihara Y,Kikugawa T,Miura N,Noda T, Kakuda T, Kitazawa R and Tanji N: Primary adrenal leiomyosarcoma with lymph node metastasis: A case report. World J Surg Oncol 14: $176,2016$.

3. Karaosmanoglu AD and Gee MS: Sonographic findings of an adrenal leiomyosarcoma. J Ultrasound Med 29: 1369-1373, 2010.

4. Nagaraj V, Mustafa M, Amin E, Ali W, Naji Sarsam S and Darwish A: Primary adrenal leiomyosarcoma in an arab male: A rare case report with immunohistochemistry study. Case Rep Surg 2015: 702541, 2015 .

5. Deshmukh SD, Babanagare SV, Anand M, Pande DP and Yavalkar P: Primary adrenal leiomyosarcoma: A case report with immunohistochemical study and review of literature. J Cancer Res Ther 9: 114-116, 2013.

6. Mohanty SK, Balani JP and Parwani AV: Pleomorphic leiomyosarcoma of the adrenal gland: Case report and review of the literature. Urology 70: 591.e5-7, 2007.
7. Mulani SR, Stoner P, Schlachterman A, Ghayee HK, Lu L and Gupte A: First reported case of endoscopic ultrasound-guided core biopsy yielding diagnosis of primary adrenal leiomyosarcoma. Case Rep Gastrointest Med 2018: 8196051, 2018.

8. Zhou Y, Tang Y, Tang J, Deng F, Gong G and Dai Y: Primary adrenal leiomyosarcoma: A case report and review of literature. Int J Clin Exp Pathol 8: 4258-4263, 2015.

9. Quildrian S, Califano I, Carrizo F, Daffinoti A and Calónico N: Primary adrenal leiomyosarcoma treated by laparoscopic adrenalectomy. Endocrinol Nutr 62: 472-473, 2015. 